\title{
Synthesis, Antibacterial and Antioxidant Activity Studies of 2, 4- Dinitrophenyl Hydrazone Derivatives of 4-Methoxyphenyl Propenone Chalcones
}

\author{
Ere $\mathrm{D}^{1^{*}}$, Eboh $\mathrm{AS}^{2}$, Ifidi $\mathrm{P}^{1}$, Dode $\mathrm{E}^{1}$ \\ ${ }^{\mathrm{T}}$ Department of Pharmaceutical and Medicinal Chemistry, Faculty of Pharmacy, Niger Delta University, Wilberforce \\ Island, Bayelsa State, Nigeria \\ ${ }^{2}$ Department of Biochemistry, Faculty of Basic Medicine, College of Health Sciences, Niger Delta University, \\ Wilberforce Island, Bayelsa State, Nigeria
}

\section{*Corresponding Author \\ Ere D}

\section{Article History}

Received: 15.09 .2020

Accepted: 24.09 .2020

Published: 30.10 .2020

\begin{abstract}
Hydrazones are an important class of biologically active molecules and could be synthesized from various methods. Similarly 2,4-dinitrophenyl hydrazine derivatives of 4-methoxyphenyl propenone chalcones are equally important hydrazones that were synthesized by the coupling of 2,4-dinitrophenyl and the 4-methoxyphenyl propenone chalcones counterparts. The coupling reaction resulted in making non - reactive 4-methoxyphenyl propenone chalcones becoming more reactive by exhibiting antibacterial, antifungal and anti-oxidant activity.
\end{abstract}

Keywords: Hydrazones, Chalcones, antibacterial, antifungal, anti-oxidant.

\section{INTRODUCTION}

Emerging bacterial resistance causes wide spread problem for the treatment of various infections, hence the search for new antimicrobials is a non - ending task and this has led to the continuous exploration and exploitation of natural products and synthetic compounds. It is to this backdrop that the researchers have decided to explore the chemistry of chalcones and its derivatives.

The study of chalcones has continued to attract attention both in academia and industry owing to its vast potential and broad spectrum of biological and pharmacological activities. Literature search reveals that chalcones biological/pharmacological activities includes oxidative, amoebicidal, anti-tubercular, anti-trichomonal, antiinflammatory, anti-malarial, anti-microbial, anti-fungal, anti-cancer etc [1,2]. Chalcones are referred as natural biocides and exhibit an array of pharmacological activities. They occur abundantly in edible plants [3] and are responsible for plant defensive mechanism to counteract reactive oxygen species in order to survive and prevent molecular damage and damage by insects and herbivores[4,5]. Chalcones are known to exist as trans and cis isomers having aromatic rings that are connected by a three carbon $\alpha, \beta$-unsaturated carbonyl system. In most cases the trans isomer are reported to be more stable from the thermodynamic perspective, which makes it the predominant configuration among chalcones. The unstable cis isomer is as a result of steric hindrance between the carbonyl group and the A ring [6]. Matos, Vazquez, Uriarte, and Santana [7], reported that chalcones are flexible molecules that exist in various conformations and their properties depend on appropriate ring substitution. Chalcones have proper electron pulling and electron pushing functional groups connected to the benzene ring(s) and can be fluorescent because of the conjugated system which makes them a potential chemical probe for mechanistic investigations.

Hydrazones are compounds that constitute an important class of biologically active drug molecules which has continued to attract the attention of medicinal chemists owing to their wide range of pharmacological properties. These compounds are being synthesized as drugs by many researchers in order to combat diseases with minimal toxicity and

Copyright (C) 2020 The Author(s): This is an open-access article distributed under the terms of the Creative Commons Attribution 4.0 International License (CC BY-NC 4.0) which permits unrestricted use, distribution, and reproduction in any medium for non-commercial use provided the original author and source are credited. 
maximal effects. This have now provided a therapeutic pathway to develop new effective biologically active compounds; hydrazones. A number of hydrazone derivatives have been reported to exert good biological activities [1, 2].

Hydrazones possess an azomethine $-\mathrm{NHN}=\mathrm{CH}$ group which are considered as derivatives of aldehydes and ketones (chalcones) in which the oxygen atom has been replaced by the $=\mathrm{NNH}_{2}$ group. Hydrazones are of wide interest because of their diverse biological applications such as anticonvulsant, antidepressant, analgesic, anti-inflammatory, antiplatelet, antimalarial, antimicrobial, antimycobacterial, anticancer, vasodilator, antiviral, anti-HIV, anthelmintic, antidiabetic, and trypanocidal activities [8 - 19].

In Ere, Dode and Usifoh [20] it was recorded that a group of 4-methoxyphenyl propenone chalcones did not exhibit any antibacterial activity. However, Fadare et al. [5] in their work "1-Indanone chalcones and their 2, 4dinitrophenyl hydrazone derivatives reported that the hydrazone derivatives had antibacterial activity. It was based on this backdrop that the group of 4-methoxyphenyl propenone chalcones that were reported not to have any antibacterial activity was coupled with 2, 4-dinitrophenyl hydrazine and the resulting hydrazones were then tested for antibacterial, antifungal and anti-oxidant activity.

\section{Materials and Methods}

Evaluation of Compounds: Structures and purity of compounds were confirmed by ${ }^{1} \mathrm{H}-\mathrm{NMR}$ using JOEL Lambda 400 spectrometer, an internal standard of TMS was used. Thin layer chromatography (TLC) (E. Merck Kieselgel 60 F254) was used to check purity of compounds. Melting point (uncorrected) was determined using Gallenkamp melting point machine.

\section{Synthesis of Chalcones}

General synthesis of 4-methoxy phenyl propanone chalcones were carried out using modified Claisen-Schmidt condensation reaction.<smiles>COc1ccc(C(=O)/C=C/c2ccco2)cc1</smiles>

3-Furan-2-yl-1-(4-methoxy-phenyl)-propenone

The compound (A) was synthesized by mixing furfural $(5.0 \mathrm{~g}, 0.05 \mathrm{~mol})$ and p-methoxyacetophenone $(7.8 \mathrm{~g}$, $0.05 \mathrm{~mol})$ in ice cold ethanol $(20 \mathrm{ml})$ in a round bottom flask equipped with magnetic stirrer for 2 hours. The temperature was monitored such that the reaction flask remained below $10{ }^{\circ} \mathrm{C}$. Ice cold $10 \%$ Potassium hydroxide $(20 \mathrm{ml})$ was added and stirred for a further 2 hours. The reaction mixture was then allowed to stand on the reaction bench at room temperature for 72 hours. To the resultant mixture was added cold distilled water $(50 \mathrm{ml})$ and then $10 \%$ acetic acid (30 $\mathrm{ml}$ ) to acidify using litmus paper to determine the acidity. The resultant crude precipitate was collected by filtration, then washed with cold water and dried. It was then recrystallized from ethanol, collected by filtration and dried in vacuo in a desiccator over silica to yield the compound A. Yield: $7.98 \mathrm{~g}, 70 \%$; mp $68-70{ }^{\circ} \mathrm{C}$; Colour: Yellow; UV: $\Lambda_{\mathrm{ma}} \mathrm{x} 260,500$, $640 \mathrm{~nm}$; IR: 2922.59, 1956.71, 1607.88, $1168.99 \mathrm{~V} \mathrm{~cm}-1$; ${ }^{1} \mathrm{H}-\mathrm{NMR}:$ [400 MHz, $\mathrm{CDCl}_{3}$ ], $\delta_{\mathrm{H}}: 3.86(3 \mathrm{H}, \mathrm{d}, \mathrm{J}=2.8 \mathrm{~Hz})$.<smiles>COc1ccc(C(=O)/C=C/c2ccccc2)cc1</smiles>

\section{1-(4-Methoxy-phenyl)-3-phenyl-propenone}

(B)

The compound (B) was synthesized by mixing p-anisaldehyde $(5.0 \mathrm{~g}, 0.04 \mathrm{~mol})$ and acetophenone $(4.41 \mathrm{~g}, 0.04$ $\mathrm{mol})$ in ice cold ethanol $(20 \mathrm{ml})$ in a round bottom flask equipped with magnetic stirrer for 2 hours. It was treated as the reaction above. Yield: $7.93 \mathrm{~g}, 90.6 \%$; mp $56-59{ }^{\circ} \mathrm{C}$; Colour: Pale Yellow; UV: $\kappa_{\max }$ 480, 580, 640, 780 nm; IR: 3734.82, 3648.69, 2923.10, 1653.21, $1601.38 \mathrm{~V} \mathrm{~cm}-1$; ${ }^{1} \mathrm{H}-\mathrm{NMR}:$ [400 MHz, $\left.\mathrm{CDCl}_{3}\right], \delta_{\mathrm{H}}:{ }^{13} \mathrm{C}-\mathrm{NMR}$, 
<smiles>COc1ccc(/C=C/C(=O)c2ccc(OC)cc2)cc1</smiles>

\section{1,3-Bis-(4-methoxy-phenyl)-propenone $\quad(\mathbf{C})$}

The compound (C) was synthesized by mixing p-anisaldehyde $(5.0 \mathrm{~g}, 0.04 \mathrm{~mol})$ and p-methoxyacetophenone $(5.51 \mathrm{~g}, 0.04 \mathrm{~mol})$ in ice cold ethanol $(20 \mathrm{ml})$ in a round bottom flask equipped with magnetic stirrer for 2 hours. Treated as in (A) above. Yield: $9.12 \mathrm{~g} \mathrm{~g}, 85 \%$; mp $68-70{ }^{\circ} \mathrm{C}$; Colour: Yellow; (Found C, 76.01; H, 6.01\%; $\mathrm{C}_{17} \mathrm{H}_{16} \mathrm{O}_{3}$ requires C, 76.10; H, 6.01\%); UV: $K_{\mathrm{ma}} \mathrm{x}$ 260, 500, $640 \mathrm{~nm}$; IR: 2922.59, 1956.71, 1607.88, $1168.99 \mathrm{~V} \mathrm{~cm}-1$; ${ }^{1} \mathrm{H}-\mathrm{NMR}:[400$ $\left.\mathrm{MHz}, \mathrm{CDCl}_{3}\right], \delta(\mathrm{ppm}): 3.75\left(3 \mathrm{H}, \mathrm{S}, \mathrm{O}-\mathrm{CH}_{3}\right), 3.84\left(3 \mathrm{H}, \mathrm{S}, \mathrm{O}-\mathrm{CH}_{3}\right), 6.77-7.05(2 \mathrm{X} 2 \mathrm{Ar}-\mathrm{H}, \mathrm{dd}), 7.66-7.68(2 \mathrm{Ar}-\mathrm{H}, \mathrm{d})$, 7.57 $(1 \mathrm{H}, \mathrm{d},=\mathrm{CH}), 7.71(1 \mathrm{H}, \mathrm{d},=\mathrm{CH}), 7.75-7.93(2 \mathrm{Xar}-\mathrm{H}, \mathrm{d}) ;{ }^{13} \mathrm{C}-\mathrm{NMR}[100 \mathrm{MHz}, \mathrm{MeOD}] \delta_{\mathrm{C}}$ 47.8, 48.0, 54.6, 113.6, $114.1,119.1,127.7,130.1,130.6,130.9,144.1,162.0,163.9,189.6 ; \mathrm{m} / \mathrm{z} 268.15\left(\mathrm{M}^{+}, 100 \%\right)$<smiles>COc1ccc(C(=O)/C=C/c2ccc(Cl)cc2)cc1</smiles>

\section{3-(4-Chloro-phenyl)-1-(4-methoxy-phenyl)-propenone}

(D)

The compound (D) was synthesized by mixing p-chlorobenzaldehyde $\left(\begin{array}{lllll}5.0 & \mathrm{~g}, & 0.04 & \mathrm{~mol}\end{array}\right)$ and pmethoxyacetophenone $(5.33 \mathrm{~g}, 0.04 \mathrm{~mol})$ in ice cold ethanol $(20 \mathrm{ml})$ in a round bottom flask equipped with magnetic stirrer for 2 hours. It was then allowed to stand at room temperature and treated as in A above. Yield: $9.42 \mathrm{~g}, 97.1 \%$; $\mathrm{mp}$ 113-115 'C; Colour: Cream; (Found C70.56, H 5.02, Cl 13.00, O 11.42; $\mathrm{C}_{16} \mathrm{H}_{13} \mathrm{ClO}_{2}$ requires C 70.46, $\mathrm{H} \mathrm{4.80,} \mathrm{Cl} \mathrm{13.00,}$ O 11.73) UV: $\kappa_{\mathrm{ma}} \mathrm{x} 380,500,640 \mathrm{~nm}$; IR: 2922.46, 2359.77, 1654.73, 1602.89, $1178.64 \mathrm{~V} \mathrm{~cm}-1$;<smiles>COc1ccc(C(=O)/C=C/c2ccc3c(c2)OCO3)cc1</smiles>

\section{3-Benzo[1,3]dioxol-5-yl-1-(4-methoxy-phenyl)- propenone}

The compound (E) was synthesized by mixing piperonal $(5.0 \mathrm{~g}, 0.03 \mathrm{~mol})$ and $\mathrm{p}$-methoxyacetophenone $(5.0 \mathrm{~g}$, $0.03 \mathrm{~mol})$ in ice cold ethanol $(20 \mathrm{ml})$ in a round bottom flask equipped with magnetic stirrer for 2 hours and was treated as A above Yield: $9.25 \mathrm{~g}, 98.4 \%$; mp 117-120 ${ }^{\circ} \mathrm{C}$; Colour: Pale-Yellow; (Found C, 72.3; H 5.02 \%; $\mathrm{C}_{17} \mathrm{H}_{14} \mathrm{O}_{4}$ requires C, 72.33; H $5.00 \%$ ), UV: $K_{\mathrm{ma}} \mathrm{x} 400,500,640,780 \mathrm{~nm}$; IR: 2922.59, 1956.71, 1607.88, $1168.99 \mathrm{~V} \mathrm{cm-1;} \mathrm{GC-MS} \mathrm{(m/z)}$ $282.11\left(\mathrm{M}^{+}, 100 \%\right), 267.09$ (14\%), 135.04 (40\%), 77 (25\%); ${ }^{1} \mathrm{H}-\mathrm{NMR}:$ [400 MHz, MeOD], $\delta(\mathrm{ppm}): 3.02(3 \mathrm{H}, \mathrm{S}$ O$\left.\mathrm{CH}_{3}\right), 6.76(1 \mathrm{H}, \mathrm{d}, \mathrm{J}=19.6 \mathrm{~Hz},=\mathrm{CH}), 7.6-7.09(\mathrm{~m}-\mathrm{H}, \mathrm{J}=3.0,15.5 \mathrm{~Hz}, 7 \mathrm{X} \mathrm{Ar}-\mathrm{H}), 8.02(1 \mathrm{H}, \mathrm{d}, \mathrm{J}=19.6 \mathrm{~Hz},=\mathrm{CH})$; 13C-NMR [MeOD, 100 MHz] $\delta$ c 38.8, 44.9, 46.9, 47.3, 47.6, 48.2, 110.8, 111.7, 115.9, 122.4, 127.9, 128.3, 130.4,<smiles>COc1ccc(C(=O)/C=C/c2ccc(Cl)cc2)cc1OC</smiles>

3-(4-Chloro-phenyl)-1-(3,4-dimethoxy-phenyl)propenone 
The compound (F) was synthesized by mixing p-chlorobenzaldehyde $\left(\begin{array}{lllll}5.0 & \mathrm{~g}, & 0.04 & \mathrm{~mol}\end{array}\right)$ and dimethoxyacetophenone $(6.37 \mathrm{~g}, 0.04 \mathrm{~mol})$ in ice cold ethanol $(20 \mathrm{ml})$ in a round bottom flask equipped with magnetic stirrer for 2 hours and then treated as sample A above. Yield: $10.21 \mathrm{~g}, 95.2 \% ; \mathrm{mp} 109-11{ }^{\circ} \mathrm{C}$; Colour: Cream; $U$ V: $\Lambda_{\mathrm{ma}} \mathrm{x}$ 500, 580640 nm; IR: 2923.48, 1668.59, $1576.67 \mathrm{~V} \mathrm{~cm}^{-1}$; m/z 302.07 (M+, $5 \%$ \%), 180 (43\%), 165 (100\%).

\section{Synthesis of hydrazones}

The scheme and table below shows the synthesis ofhydrazones.

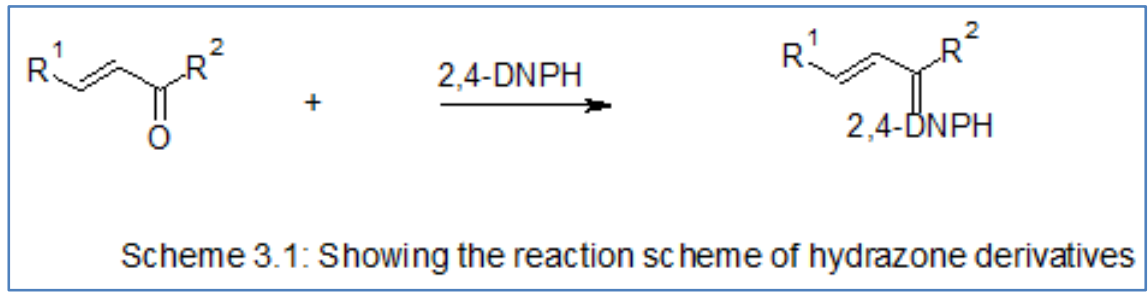

Table-1: Showing reagents used and corresponding hydrazone products.

\begin{tabular}{|c|c|c|c|}
\hline $\mathbf{R}_{1}$ & $\mathbf{R}_{2}$ & Structure and name of compound & Physical properties/Code \\
\hline Furan & 4-Methoxy phenyl & (E)-1-(2,4-dinitrophenyl)-2-((E)-3-(furan-2- & 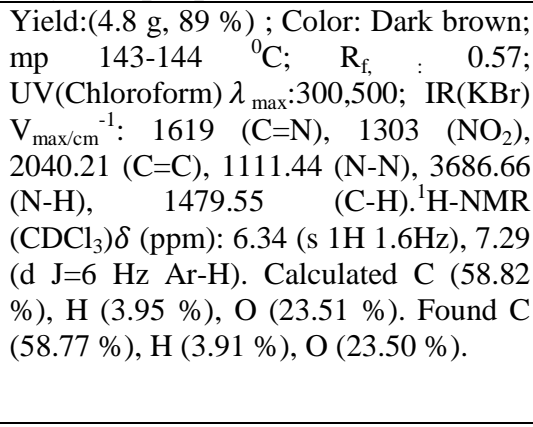 \\
\hline Phenyl & Benzo[d][1,3]dioxole & $\mathrm{NO}_{2}$ & $\begin{array}{l}\text { Yield:(3.4 g, } 66.6 \%) \text {; Color: Reddish } \\
\text { precipitate; mp } 189-191{ }^{\circ} \mathrm{C} ; \mathrm{R}_{\mathrm{f}}: 0.64 ; \\
\text { UV(Chloroform) } \lambda \max : 200,250, \\
300,400 \mathrm{~nm} \text {; IR }(\mathrm{KBr}) \mathrm{V}_{\max / \mathrm{cm}^{-1}}: 1661 \\
(\mathrm{C}=\mathrm{N}), 1545(-\mathrm{Ar}), 3367(\mathrm{NH}), 1380.34 \\
\left(\mathrm{NO}_{2}\right), 1147.61(\mathrm{~N}-\mathrm{N}), 1712 \quad(\mathrm{C}=\mathrm{C}), \\
1452(\mathrm{C}-\mathrm{H}) .{ }^{1} \mathrm{H}-\mathrm{NMR}\left(\mathrm{CDCl}_{3}\right) \delta(\mathrm{ppm}) \text { : } \\
1.17(1 \mathrm{H} 1.2 \mathrm{~Hz}), 4.07(1 \mathrm{H} 2.3 \mathrm{~Hz}), 6.63 \\
(\mathrm{Ar}-12 \mathrm{H}) \text { Calculated C }(61.11 \%), \mathrm{H} \\
(3.73 \%), \mathrm{O}(22.20 \%) . \text { Found } \mathrm{C}(61.06 \\
\%), \mathrm{H}(3.75 \%), \mathrm{O}(22.50 \%) .\end{array}$ \\
\hline $\begin{array}{l}\text { 4- } \\
\text { Chlorophenyl }\end{array}$ & 4-Methoxy phenyl & $\mathrm{NO}_{2}$ & 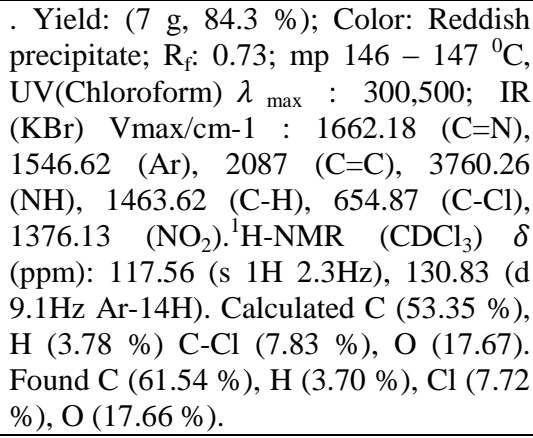 \\
\hline $\begin{array}{l}\text { 4-Methoxy } \\
\text { phenyl }\end{array}$ & 4-Methoxy phenyl & $\begin{array}{l}\mathrm{CH}_{3} \\
\text { O }\end{array}$ & 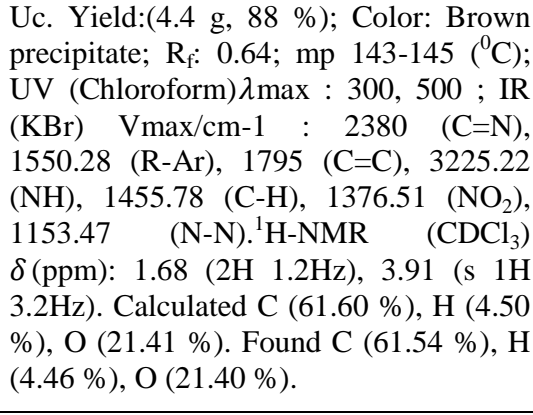 \\
\hline
\end{tabular}




\begin{tabular}{|c|c|c|c|}
\hline $\begin{array}{l}\text { 4-Chloro } \\
\text { phenyl }\end{array}$ & $\begin{array}{l}\text { 3,4-dimethoxy } \\
\text { phenyl }\end{array}$ & $\begin{array}{l}\text { (E)-1-((E)-3-(4-chlorophenyl)-1-(3,4- } \\
\text { dimethoxyphenyl)allylidene)-2-(2,4- } \\
\text { dinitrophenyl)hydrazine }\end{array}$ & 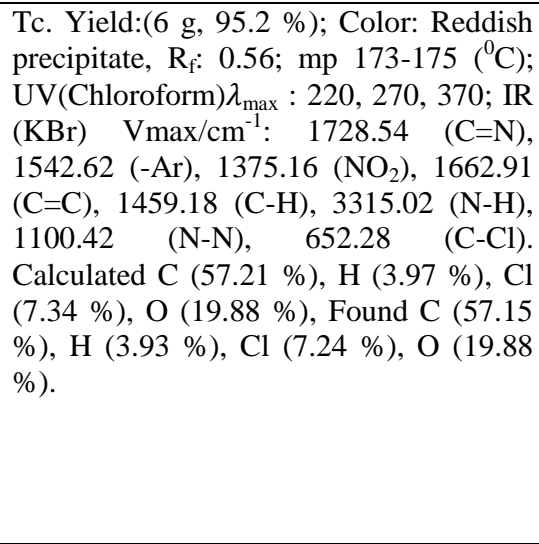 \\
\hline Phenyl & 4-Methoxy phenyl & $\begin{array}{l}\text { (E)-1-(2,4-dinitrophenyl)-2-((E)-1-(4- } \\
\text { methoxyphenyl)-3- } \\
\text { phenylallylidene)hydrazine }\end{array}$ & $\begin{array}{l}\text { Rc. Yield (5.4 g, 77.1 \%); Color Reddish } \\
\text { precipitate; } \mathrm{R}_{\mathrm{f}}: 0.7, \mathrm{mp} 148-150\left({ }^{0} \mathrm{C}\right) ; \mathrm{UV} \\
\text { (Chloroform) } \lambda \max : 280,480 . \mathrm{IR}(\mathrm{KBr}) \\
\mathrm{Vmax} / \mathrm{cm}^{-1}: 1729.96(\mathrm{C}=\mathrm{N}), 1604.70(- \\
\mathrm{Ar}), 1503.32\left(\mathrm{NO}_{2}\right), 1801.79(\mathrm{C}=\mathrm{C}), \\
3261.02(\mathrm{NH}), 1432.15(\mathrm{C}-\mathrm{H}), 1118.01 \\
(\mathrm{~N}-\mathrm{N}) .{ }^{1} \mathrm{H}-\mathrm{NMR}\left(\mathrm{CDCl}_{3}\right) \delta(\mathrm{ppm}): 3.96 \\
(1 \mathrm{H} 1.8 \mathrm{~Hz}), 6.64(\mathrm{~d} 6.2 \mathrm{~Hz} \mathrm{Ar}-13 \mathrm{H}) . \\
\text { Calculated C }(63.15 \%), \mathrm{H}(4.34 \%), \mathrm{O} \\
(19.12 \%) . \text { Found C }(63.09 \%), \mathrm{H}(4.30 \\
\%), \mathrm{O}(19.12 \%) .\end{array}$ \\
\hline $\begin{array}{l}\text { Benzo[d]1,3- } \\
\text { dioxole }\end{array}$ & 4-methoxy phenyl & $\begin{array}{l}\text { (E)-1-((E)-3-(benzo[d][1,3]dioxol-5-yl)-1-(4- } \\
\text { methoxyphenyl)allylidene)-2-(2,4- } \\
\text { dinitrophenyl)hydrazine }\end{array}$ & $\begin{array}{l}\text { Xc. Yield: }(5 \mathrm{~g}, 76.9 \%) \text {; Color: Brown } \\
\text { precipitate; } \mathrm{R}_{\mathrm{f}}: 0.61 ; \mathrm{mp} 150-152\left({ }^{0} \mathrm{C}\right) \text {; } \\
\mathrm{UV}(\text { Chloroform }) \lambda \max : 210,380 ; \mathrm{IR} \\
(\mathrm{KBr}) \mathrm{Vmax} / \mathrm{cm}^{-1}: 1830.17 \quad(\mathrm{C}=\mathrm{N}) \text {, } \\
1601.55(-\mathrm{Ar}), 1497.49\left(\mathrm{NO}_{2}\right), 1445.51 \\
(\mathrm{C}-\mathrm{H}), 2044.47(\mathrm{C}=\mathrm{C}), 3278.22(\mathrm{NH}) \text {. } \\
\text { Calculated C }(59.74 \%), \mathrm{H}(3.92 \%), \mathrm{O} \\
(24.22 \%) \text {. Found C }(59.68 \%), \mathrm{H}(3.89 \\
\%), \mathrm{O}(24.22 \%) .\end{array}$ \\
\hline $\begin{array}{l}\text { 4-Chloro } \\
\text { phenyl }\end{array}$ & Phenyl & $\begin{array}{l}\text { (E)-1-((E)-3-(4-chlorophenyl)-1- } \\
\text { phenylallylidene)-2-(2,4- } \\
\text { dinitrophenyl)hydrazine }\end{array}$ & 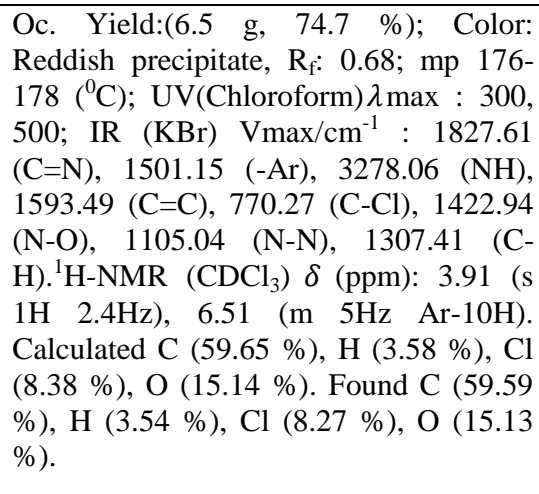 \\
\hline
\end{tabular}

\section{Antibacterial Activity}

The test organisms were clinical isolates obtained from the Department of Medical Microbiology, College of Health Sciences, Niger Delta University Wilberforce Island Amassoma Bayelsa State Nigeria. Baclllussubstilis (Gram positive) Escherichia coli (Gram negative), Staphylococcus aureus (Gram positive), Pseudomonas species (Gram negative), Proteus species (Gram negative) and Candida albican were used. These organisms were stored in a Cryopreservation medium prior to the experiment. 


\section{Preparation of standard of synthesized compound}

The synthesized compounds (100 mg) were weighed and dissolved in DMSO $10 \mathrm{ml}(10 \mathrm{mg} / \mathrm{ml})$. McCartney bottles were sterilized and labeled accordingly. The various respective concentrations of the synthesized compounds were prepared and transferred into the bottles.

\section{Evaluation of antimicrobial activity}

Agar dilution method using Mueller Hintonagar (Merck, Germany) medium was used to evaluate the antimicrobial effects of the dimethylsulfoxide (DMSO) solvent. Mueller Hintonagar medium was prepared sterile, cooled to $45{ }^{\circ} \mathrm{C}$ and was poured on the sterile plates and allowed to set. Media plates were then seeded with the test isolates, holes were bored in the culture medium with the aid of a cork borer of $11 \mathrm{~mm}$ in diameter by sterilizing the cork borer with a spirit lamp after each hole was bored. Molten agar was used after to seal the bottom of the holes to prevent leakage. The prepared compounds and standard antimicrobial disc of known concentrations were placed in culture medium using a pipette and disc dispenser. Plates were then incubated inverted at $37{ }^{\circ} \mathrm{C}$ for $18-24$ hrs and the diameter of freezone was measured exactly by using a ruler in millimeters.

\section{Evaluation of antimicrobial minimum inhibitory concentration (MIC)}

Serial dilutions were made from the original $10 \mathrm{mg} / \mathrm{ml}$ concentration to $5 \mathrm{mg} / \mathrm{ml}, 2.5 \mathrm{mg} / \mathrm{ml} \mathrm{and} 1 \mathrm{mg} / \mathrm{ml} \mathrm{of} \mathrm{the}$ various hydrazones in DMSO at $0.4 \mathrm{ml}$ drop and the minimum inhibitory concentration was determined.

\section{Antioxidant Activity}

The coupled products were dissolved in DMSO at various concentrations of $0.01 \mathrm{~g} / 10 \mathrm{ml}, 0.03 \mathrm{~g} / 10 \mathrm{ml}, 0.05$ $\mathrm{g} / 10 \mathrm{ml}$ and $0.07 \mathrm{~g} / 10 \mathrm{ml}$. Ascorbic acid was used as a reference standard. $0.006 \mathrm{~g}$ of 1,1-diphenyl-2-picrylhydrazyl (DPPH) was freshly prepared in ethanol $(20 \mathrm{ml}) .500 \mu \mathrm{l}$ of DPPH was added to each test tube containing various concentrations of the hydrazones and standard solution, and was shaking vigorously. The mixture was then left in the dark for 30 minutes. The control was carried out without the addition of DPPH and the hydrazones and the absorbance were taken at $516 \mathrm{~nm}$ in UV-Visible spectrophotometer. The scavenging activity was determined as a percentage of the DPPH free radical activity with the formula \% RSA = (DPPH - COMPOUND $) / D P P H$.

\section{RESULTS AND DISCUSSION}

Table-2: Zone of inhibition (ZOI) of each synthesized compound at $10 \mathrm{mg} / \mathrm{ml}$

\begin{tabular}{|c|c|c|c|c|c|c|}
\hline \multirow[t]{3}{*}{ Sample } & \multicolumn{6}{|c|}{ Zone of inhibition (mm) } \\
\hline & \multicolumn{6}{|l|}{ Isolate } \\
\hline & $\begin{array}{l}\text { Bacillus } \\
\text { subtillis }\end{array}$ & $\begin{array}{l}\text { Echerichia } \\
\text { coli }\end{array}$ & $\begin{array}{l}\text { Candida } \\
\text { albican }\end{array}$ & $\begin{array}{l}\text { Proteus } \\
\text { species }\end{array}$ & $\begin{array}{l}\text { Staphylococcus } \\
\text { aureus }\end{array}$ & $\begin{array}{l}\text { Pseudomonas } \\
\text { species }\end{array}$ \\
\hline $\mathrm{Bc}$ & -- & -- & -- & -- & -- & 22 \\
\hline$\overline{\mathrm{Pc}}$ & 20 & -- & -- & 17 & 20 & 20 \\
\hline $\mathrm{Wc}$ & 22 & -- & -- & -- & -- & 11 \\
\hline $\mathrm{Uc}$ & 14 & -- & -- & -- & -- & 11 \\
\hline $\mathrm{Tc}$ & -- & -- & -- & -- & 20 & 16 \\
\hline $\mathrm{Rc}$ & -- & -- & -- & -- & -- & 20 \\
\hline $\mathrm{Xc}$ & -- & -- & -- & -- & -- & 21 \\
\hline $\mathrm{Oc}$ & 15 & 19 & -- & 10 & -- & 18 \\
\hline AML & 20 & -- & -- & -- & 22 & 26 \\
\hline CIPRO & 32 & 28 & -- & 30 & 31 & 28 \\
\hline ERY & -- & 17 & -- & -- & 10 & 24 \\
\hline DMSO & -- & -- & -- & -- & -- & -- \\
\hline
\end{tabular}

Key: -- No zone of inhibition; AML Amoxicillin; CIPRO Ciprofloxacin; ERY Erythromycin; DMSO Dimethylsulfoxide 
Table-3

\begin{tabular}{|c|c|c|c|c|c|c|c|}
\hline \multirow[b]{3}{*}{ Samples } & \multicolumn{7}{|c|}{ Minimum inhibitory concentrations (MICs) } \\
\hline & \multicolumn{7}{|c|}{ Isolates } \\
\hline & $\begin{array}{l}\text { Concentration } \\
(\mathrm{mg} / \mathrm{ml})\end{array}$ & $\begin{array}{l}\text { Bacillus } \\
\text { subtilis }\end{array}$ & $\begin{array}{l}\text { Echerichia } \\
\text { coli }\end{array}$ & $\begin{array}{l}\text { Candida } \\
\text { albican }\end{array}$ & $\begin{array}{l}\text { Proteus } \\
\text { spp }\end{array}$ & $\begin{array}{l}\text { Staphylococcus } \\
\text { aureus }\end{array}$ & $\begin{array}{l}\text { Pseudomonas } \\
\text { aeruginosa }\end{array}$ \\
\hline $\mathrm{Bc}$ & $\begin{array}{l}5 \\
2.5 \\
1\end{array}$ & $\begin{array}{l}-- \\
-- \\
--\end{array}$ & $\begin{array}{l}- \\
-- \\
--\end{array}$ & $\begin{array}{l}-- \\
-- \\
--\end{array}$ & $\begin{array}{l}-- \\
-- \\
--\end{array}$ & $\begin{array}{l}-- \\
-- \\
--\end{array}$ & $\begin{array}{l}+ \\
+ \\
+\end{array}$ \\
\hline $\mathrm{Pc}$ & $\begin{array}{l}5 \\
2.5 \\
1 \\
\end{array}$ & $\begin{array}{l}+ \\
+ \\
+\end{array}$ & $\begin{array}{l}-- \\
-- \\
--\end{array}$ & $\begin{array}{l}-- \\
-- \\
--\end{array}$ & $\begin{array}{l}-- \\
-- \\
--\end{array}$ & $\begin{array}{l}+ \\
+ \\
+\end{array}$ & $\begin{array}{l}+ \\
+ \\
+\end{array}$ \\
\hline Wc & $\begin{array}{l}5 \\
2.5 \\
1 \\
\end{array}$ & $\begin{array}{l}+ \\
+ \\
+\end{array}$ & $\begin{array}{l}- \\
-- \\
--\end{array}$ & $\begin{array}{l}-- \\
-- \\
--\end{array}$ & $\begin{array}{l}+ \\
+ \\
+\end{array}$ & $\begin{array}{l}- \\
-- \\
--\end{array}$ & $\begin{array}{l}+ \\
+ \\
+\end{array}$ \\
\hline Uc & $\begin{array}{l}5 \\
2.5 \\
1 \\
\end{array}$ & $\begin{array}{l}+ \\
+ \\
+\end{array}$ & $\begin{array}{l}- \\
-- \\
--\end{array}$ & $\begin{array}{l}- \\
-- \\
--\end{array}$ & $\begin{array}{l}- \\
-- \\
--\end{array}$ & $\begin{array}{l}- \\
-- \\
--\end{array}$ & $\begin{array}{l}+ \\
+ \\
+\end{array}$ \\
\hline Tc & $\begin{array}{l}5 \\
2.5 \\
1 \\
\end{array}$ & $\begin{array}{l}-- \\
-- \\
-- \\
\end{array}$ & $\begin{array}{l}-- \\
-- \\
--\end{array}$ & $\begin{array}{l}-- \\
-- \\
--\end{array}$ & $\begin{array}{l}-- \\
-- \\
--\end{array}$ & $\begin{array}{l}+ \\
+ \\
--\end{array}$ & $\begin{array}{l}+ \\
+ \\
+\end{array}$ \\
\hline $\mathrm{Rc}$ & $\begin{array}{l}5 \\
2.5 \\
1 \\
\end{array}$ & $\begin{array}{l}-- \\
-- \\
--\end{array}$ & $\begin{array}{l}- \\
-- \\
--\end{array}$ & $\begin{array}{l}-- \\
-- \\
--\end{array}$ & $\begin{array}{l}- \\
-- \\
--\end{array}$ & $\begin{array}{l}- \\
-- \\
--\end{array}$ & $\begin{array}{l}+ \\
+ \\
+\end{array}$ \\
\hline $\mathrm{Xc}$ & $\begin{array}{l}5 \\
2.5 \\
1 \\
\end{array}$ & $\begin{array}{l}-- \\
-- \\
-- \\
\end{array}$ & $\begin{array}{l}-- \\
-- \\
-- \\
\end{array}$ & $\begin{array}{l}-- \\
-- \\
--\end{array}$ & $\begin{array}{l}-- \\
-- \\
-- \\
\end{array}$ & $\begin{array}{l}-- \\
-- \\
-- \\
\end{array}$ & $\begin{array}{l}+ \\
+ \\
+\end{array}$ \\
\hline Oc & $\begin{array}{l}5 \\
2.5 \\
1\end{array}$ & $\begin{array}{l}+ \\
-- \\
--\end{array}$ & $\begin{array}{l}+ \\
+ \\
+\end{array}$ & $\begin{array}{l}-- \\
-- \\
--\end{array}$ & $\begin{array}{l}+ \\
+ \\
+\end{array}$ & $\begin{array}{l}- \\
-- \\
--\end{array}$ & $\begin{array}{l}+ \\
+ \\
+\end{array}$ \\
\hline AML & $\operatorname{disc}(10 \mu g)$ & + & + & -- & + & + & + \\
\hline CIPRO & $\operatorname{disc}(10 \mu g)$ & + & + & -- & + & + & + \\
\hline ERY & $\operatorname{disc}(10 \mu g)$ & + & + & -- & + & -- & + \\
\hline
\end{tabular}

Key: -- No growth; + Growth

Table-4: Antioxidant activity of synthesized compounds by DPPH method.

\begin{tabular}{|c|c|c|c|c|}
\hline \multirow[t]{2}{*}{ Compounds } & \multicolumn{4}{|c|}{ Scavenging Effect $(\% @ \mu \mathrm{g} / \mathrm{mL})$} \\
\hline & $1 \mu \mathrm{g} / \mathrm{ml}$ & $3 \mu \mathrm{g} / \mathrm{ml}$ & $5 \mu \mathrm{g} / \mathrm{ml}$ & $7 \mu \mathrm{g} / \mathrm{ml}$ \\
\hline $\mathrm{Bc}$ & $73.35 \pm 0.016$ & $73.34 \pm 0.016$ & $76.43 \pm 0.016$ & $78.35 \pm 0.016$ \\
\hline Pc & $40.79 \pm 0.087$ & $64.35 \pm 0.087$ & $66.12 \pm 0.087$ & $68.48 \pm 0.087$ \\
\hline $\mathrm{Wc}$ & $-17.52 \pm 0.033$ & $-12.51 \pm 0.033$ & $-10.01 \pm 0.033$ & $-5.89 \pm 0.033$ \\
\hline Uc & $62.44 \pm 0.038$ & $67.45 \pm 0.038$ & $54.49 \pm 0.038$ & $65.25 \pm 0.038$ \\
\hline Tc & $87.03 \pm 0.007$ & $87.92 \pm 0.007$ & $87.33 \pm 0.007$ & $89.39 \pm 0.007$ \\
\hline $\mathrm{Rc}$ & $62.16 \pm 0.049$ & $64.65 \pm 0.049$ & $70.69 \pm 0.049$ & $76.14 \pm 0.049$ \\
\hline $\mathrm{Xc}$ & $74.22 \pm 0.062$ & $77.76 \pm 0.062$ & $90.27 \pm 0.062$ & $92.78 \pm 0.062$ \\
\hline Oc & $63.91 \pm 0.042$ & $68.77 \pm 0.042$ & $73.93 \pm 0.042$ & $78.20 \pm 0.042$ \\
\hline Ascorbic acid & $88.80 \pm 0.018$ & $88.51 \pm 0.018$ & $92.63 \pm 0.018$ & $93.81 \pm 0.018$ \\
\hline
\end{tabular}

The chalcones were synthesized following Claisen-Schmidt condensation reaction of aromatic aldehyde (Benzaldehyde) with an appropriate acetophenone in the presence of aqueous alkali at low temperature. The corresponding hydrazones were achieved when the chalcones were coupled with 2,4-DNPH in ethanol using concentrated sulfuric acid as a catalyst. The reaction mechanism is perceived to have followed the scheme as shown below. 


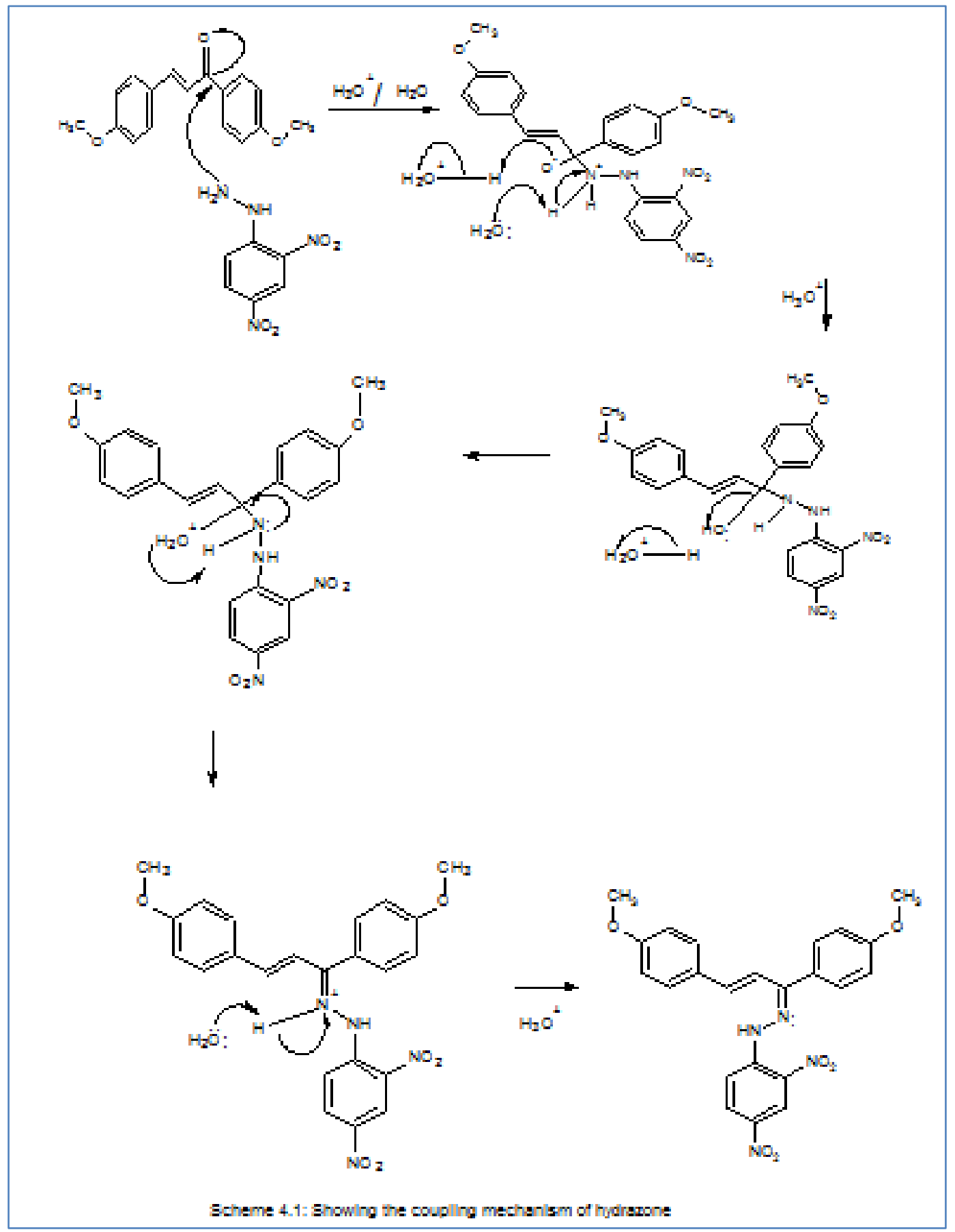

The coupling with 2,4-DNPH was relatively quick, lasting just about 20 minutes maximum as confirmed by TLC and the yields were good, between $(66.6-95.2 \%)$.

The various hydrazones obtained gave melting points ranging from 144 to $191{ }^{\circ} \mathrm{C}$ and it was observed that they all gave bright colors ranging from yellow to red. The $\lambda_{\max }$ for each of the synthesized hydrazones was determined and the values are shown in the spectra table. Compound Pc has $\lambda_{\max }$ at $300,450 \mathrm{~nm}$ and the corresponding chalcone moiety recorded its $\lambda_{\max }$ at $500,580 \mathrm{~nm}$ indicating a hypsochromic shift. This shift could be attributed to the auxochromes attached to the imine conjugate as a result of the 2, 4-DNPH coupling.

From the IR spectra, the hydrazones recorded absorption at $1475.55 \mathrm{~cm}^{-1}$ and $1560 \mathrm{~cm}^{-1}$ relating to compound $\mathrm{Bc}$, which indicates the presence of aromatic and imine functional groups. The imine $(-\mathrm{HC}=\mathrm{N})$ functional group was absent in the chalcone moiety and it shows a strong peak at $1640-1680 \mathrm{~cm}^{-1}$ in the corresponding hydrazone product which indicates that the coupling was successful. The aromatic rings of (1Z)-1-[(2E)-3-(4-chlorophenyl)-1-(4- 
methoxyphenyl)prop-2-en-1-ylidene]-2-(2,4,dinitrophenyl)hydrazine[Wc] shows a sharp peak of medium intensity at $1546.63 \mathrm{~cm}^{-1}$ pointing towards the presence of aromatic compounds and these may just affirm that coupling took place.

${ }^{1} \mathrm{H}-\mathrm{NMR}$ of the hydrazones was recorded using deuterated chloroform with TMS (tetramethylsilane) as internal standard. The chemical shifts are expressed as $\delta$ values (ppm). The ${ }^{1} \mathrm{H}-\mathrm{NMR}$ spectra of hydrazones shows a cluster of signals around the aromatic region $6.60-8.40 \mathrm{ppm}$ and this may signify the presence of three aromatic rings as the total number of proton was found to be nineteen (19) in (1Z)-1-[(2E)-3-(4-chlorophenyl)-1-phenylprop-2-en-1-ylidene]-2(2,4-dinitrophenyl)hydrazine [Oc]. (2Z)-1-(2,4-dinitrophenyl)-2-[(2E)-1-(furan-2-yl)-3-(4-methoxyphenyl)prop-2-en-1ylidene]hydrazine $[\mathrm{Bc}]$ on the other hand showed a highly deshielded methyl peak at $1.4 \mathrm{ppm}$ indicating the presence of methoxy group.

From the spectra analysis of the ${ }^{13} \mathrm{C}$ NMR, peaks were observed at $40-52 \mathrm{ppm}$. The peak observed between 120 - 154 ppm showed twenty three (23) numbers of carbons indicates the presence of aromatic carbon in compound Bc. The presence of methoxy group indicates that a methyl carbon is attached to an electronegative element which could cause a shielding effect in compound Wc.

The antimicrobial activities of the hydrazones were carried out using the agar-well diffusion method against two strains of Gram-positive, three Gram-negative bacteria and one Candida albican. Antimicrobial susceptibility of the eight (8) compounds was tested by determining the zone of inhibition and the minimum inhibitory concentrations, whose values are summarized in Tables above. The standard drugs used were Amoxicillin, Ciprofloxacin and Erythromycin, and the activity of all the coupled hydrazones were less in comparison to the standard drugs. The hydrazonecompounds (2Z)-1-(2,4-dinitrophenyl)-2-[(2E)-1-(furan-2-yl)-3-(4-methoxyphenyl)prop-2-en-1-ylidene]hydrazine[Bc] and (1Z)-1[(2E)-3-(4-chlorophenyl)-1-(4-methoxyphenyl)prop-2-en-1-ylidene]-2-(2,4,dinitrophenyl)hydrazine[Wc] having furan and 4-chlorophenyl-1-4-methoxyphenyl in their respective hydrazone framework were found to be the most active against Gram-negative Pseudomonas species and Gram-positive Bacillus subtillis bacterial strains. Compound Xc having 3-(benzol[d][1,3]dioxol-5-yl)-1-(4-methoxyphenyl) and Pc having -3-(1,3-benzodioxol-5-yl) in their hydrazone structures were next in potency against Gram-negative Pseudomonas species and Gram-positive Bacillus subtillis strains of bacteria.

Compound Tc and Oc with chlorine substituent in their hydrazone moiety also have a lesser susceptibility on Gram-positive Staphylococcus aureus and Gram-negative Echerichia coli and Proteus spp bacterial strains .None was susceptible to the Candida albican.

Minimum inhibitory concentration (MIC) was further carried out in agar diffusion medium at concentrations of $5 \mathrm{mg} / \mathrm{ml}, 2.5 \mathrm{mg} / \mathrm{ml}$ and $1 \mathrm{mg} / \mathrm{ml}$, and compound Tc has a minimum inhibitory activity on Staphylococcus aureus at a concentration of $5 \mathrm{mg} / \mathrm{ml}$. These indicates that when chalcones are coupled with 2,4-DNPH to form hydrazones, antibacterial activity is certain. This is contrary to Ere, Dode and Usifoh [17] report which states that the synthesized 4methoxyphenylpropenone (chalcones) does not have antibacterial activity.

There are numerous antioxidant assays in literature with different methodologies but the 1,1-diphenyl-2picrylhydrazyl (DPPH) radical scavenging activity was used. The antioxidant activities of the hydrazones were evaluated using DPPH radical scavenging method. Ascorbic acid was used as a positive control. The DPPH radical scavenging activity was carried out at concentration of $0.01 \mathrm{~g} / 10 \mathrm{ml}$ and the results were reported in three replicates. The absorbance which was taken at $516 \mathrm{~nm}$ showed a significant relationship between percentage concentration/inhibitions of hydrazones. The DPPH radical scavenging activity was expressed as percentage (\%) of inhibition and results are tabulated in Table 4.4. Among the derived hydrazones, compound Xc gave $(92.78 \%)$ while Tc and Bc gave (89.39 \%) and $(78.35 \%)$ respectively which exhibited maximum DPPH free radical scavenging activity followed by compound Oc, $\mathrm{Pc}$, and Rc which also gave $(78.20 \%),(68.48 \%)$, (76.14 \%) respectively, and these compounds have lesser radical scavenging activity when compared with the reference standard ascorbic acid which gave (93.81 \%). The lowest scavenging activity was observed in compounds Uc and WC which gave $(65.25 \%)$ and $(-5.89 \%)$. The investigation of antioxidant screening revealed that some of the tested compounds show moderate to good antioxidant activity. This could be attributed to the positioning of the benzene rings in the structures of the various chalcones conferring some antioxidant activity based on resonance stability. The antioxidant activity could also be due to the activated benzene ring making it to scavenge more free radicals than inactivated rings in the chalcones.

\section{REFERENCES}

1. Oyedapo, O. O., Akinpelu, B. A., \& Orefuwa, S. O. (2004). Anti-inflammatory effect of Theobroma cacao, root extract. J Trop Med Plants, 5(2), 161-6.

2. Go, M. L., Wu, X., \& Liu, X. L. (2005). Chalcones: an update on cytotoxic and chemoprotective properties. Current medicinal chemistry, 12(4), 483-499. 
3. Chavan, B. B., Gadekar, A. S., Mehta, P. P., Vawhal, P. K., Kolsure, A. K., \& Chabukswar, A. R. (2016). Synthesis $\&$ Medicinal Significance of Chalcones-A Review. Asian Journal of Biomedical and Pharmaceutical Sciences, 6(56), 01.

4. Vaya, J., Belinky, P. A., \& Aviram, M. (1997). Antioxidant constituents from licorice roots: isolation, structure elucidation and antioxidative capacity toward LDL oxidation. Free Radical Biology and Medicine, 23(2), $302-313$.

5. Fadare, O. A., Akinpelu, D. A., Ejemubu, H., \& Obafemi, C. A. (2014). 1-Indanone chalcones and their 2, 4Dinitrophenylhydrazone derivatives: Synthesis, physicochemical properties and in vitro antibacterial activity. African Journal of Pure and Applied Chemistry, 8(4), 68-77.

6. Marcelo. (2017).

7. Matos. (2017).

8. Lin, Y. M., Zhou, Y., Flavin, M. T., Zhou, L. M., Nie, W., \& Chen, F. C. (2002). Chalcones and flavonoids as antituberculosis agents. Bioorganic \& medicinal chemistry, 10(8), 2795-2802.

9. Nowakowska, Z. (2007). A review of anti-infective and anti-inflammatory chalcones. European journal of medicinal chemistry, 42(2), 125-137.

10. Velavan, S., Nagulendran, K., Mahesh, R., \& Begum, V. H. (2007). In vitro antioxidant activity of Asparagus racemosus root. Pharmacognosy magazine, 3(9), 26.

11. Seleem, H. S., El-Inany, G. A., El-Shetary, B. A., Mousa, M. A., \& Hanafy, F. I. (2011). The ligational behavior of an isatinic quinolyl hydrazone towards copper (II)-ions. Chemistry Central Journal, 5(1), 20.

12. Abdel-Wahab, B. F., Awad, G. E., \& Badria, F. A. (2011). Synthesis, antimicrobial, antioxidant, anti-hemolytic and cytotoxic evaluation of new imidazole-based heterocycles. European journal of medicinal chemistry, 46(5), 15051511.

13. Abu-Surrah, A. S., Safieh, K. A. A., Ahmad, I. M., Abdalla, M. Y., Ayoub, M. T., Qaroush, A. K., \& AbuMahtheieh, A. M. (2010). New palladium (II) complexes bearing pyrazole-based Schiff base ligands: Synthesis, characterization and cytotoxicity. European journal of medicinal chemistry, 45(2), 471-475.

14. Ajani, O. O., Obafemi, C. A., Nwinyi, O. C., \& Akinpelu, D. A. (2010). Microwave assisted synthesis and antimicrobial activity of 2-quinoxalinone-3-hydrazone derivatives. Bioorganic \& medicinal chemistry, 18(1), 214221.

15. Al-Said, M. S., Bashandy, M. S., Al-Qasoumi, S. I., \& Ghorab, M. M. (2011). Anti-breast cancer activity of some novel 1, 2-dihydropyridine, thiophene and thiazole derivatives. European Journal of Medicinal Chemistry, 46(1), $137-141$

16. Aslam, M. A. S., Mahmood, S. U., Shahid, M., Saeed, A., \& Iqbal, J. (2011). Synthesis, biological assay in vitro and molecular docking studies of new Schiff base derivatives as potential urease inhibitors. European Journal of Medicinal Chemistry, 46(11), 5473-5479.

17. Cui, Z., Li, Y., Ling, Y., Huang, J., Cui, J., Wang, R., \& Yang, X. (2010). New class of potent antitumor acylhydrazone derivatives containing furan. European journal of medicinal chemistry, 45(12), 5576-5584.

18. Kaushik, D., Khan, S. A., Chawla, G., \& Kumar, S. (2010). N'-[(5-chloro-3-methyl-1-phenyl-1H-pyrazol-4-yl) methylene] 2/4-substituted hydrazides: Synthesis and anticonvulsant activity. European Journal of Medicinal Chemistry, 45(9), 3943-3949.

19. Edrees, M. M., Farghaly, T. A., El-Hag, F. A., \& Abdalla, M. M. (2010). Antimicrobial, antitumor and 5 $\alpha$-reductase inhibitor activities of some hydrazonoyl substituted pyrimidinones. European journal of medicinal chemistry, 45(12), 5702-5707. 